\title{
LA-UR-18-29577
}

Approved for public release; distribution is unlimited.

Title: $\quad$ Smart Monitoring of Nuclear Facilities

Author(s): $\quad$ Miller, Karen Ann

Intended for: University Lecture

Issued: 2018-10-09 
Disclaimer:

Los Alamos National Laboratory, an affirmative action/equal opportunity employer, is operated by the Los Alamos National Security, LLC for the National Nuclear Security Administration of the U.S. Department of Energy under contract DE-AC52-06NA25396. By approving this article, the publisher recognizes that the U.S. Government retains nonexclusive, royalty-free license to publish or reproduce the published form of this contribution, or to allow others to do so, for U.S. Government purposes. Los Alamos National Laboratory requests that the publisher identify this article as work performed under the auspices of the U.S. Department of Energy. Los Alamos National Laboratory strongly supports academic freedom and a researcher's right to publish; as an institution, however, the Laboratory does not endorse the viewpoint of a publication or guarantee its technical correctness. 


\section{Smart Monitoring of Nuclear Facilities}

October 2018

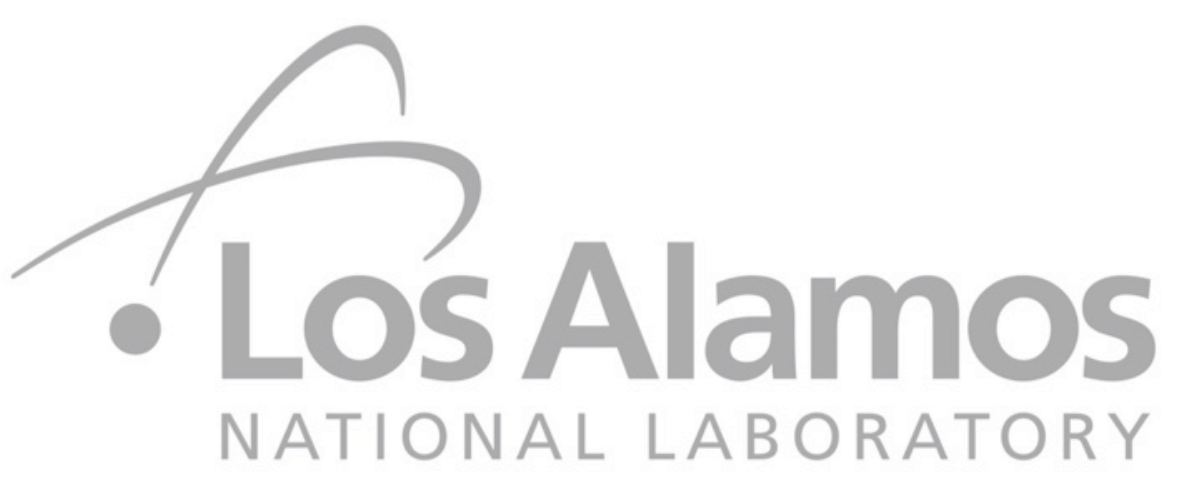

Dr. Karen Miller

Safeguards Science \& Technology Group

NAS蹨 


\section{Introduction}

This project pulls inspiration from modern smart home technologies and commercial loT sensing and applies it to the challenge of monitoring and verification of nuclear facilities

Building off the idea that the whole is greater than the sum of its parts, the goal is to develop methods for combining disparate data representative of typical IAEA data streams to improve detection of undeclared activities at safeguarded facilities

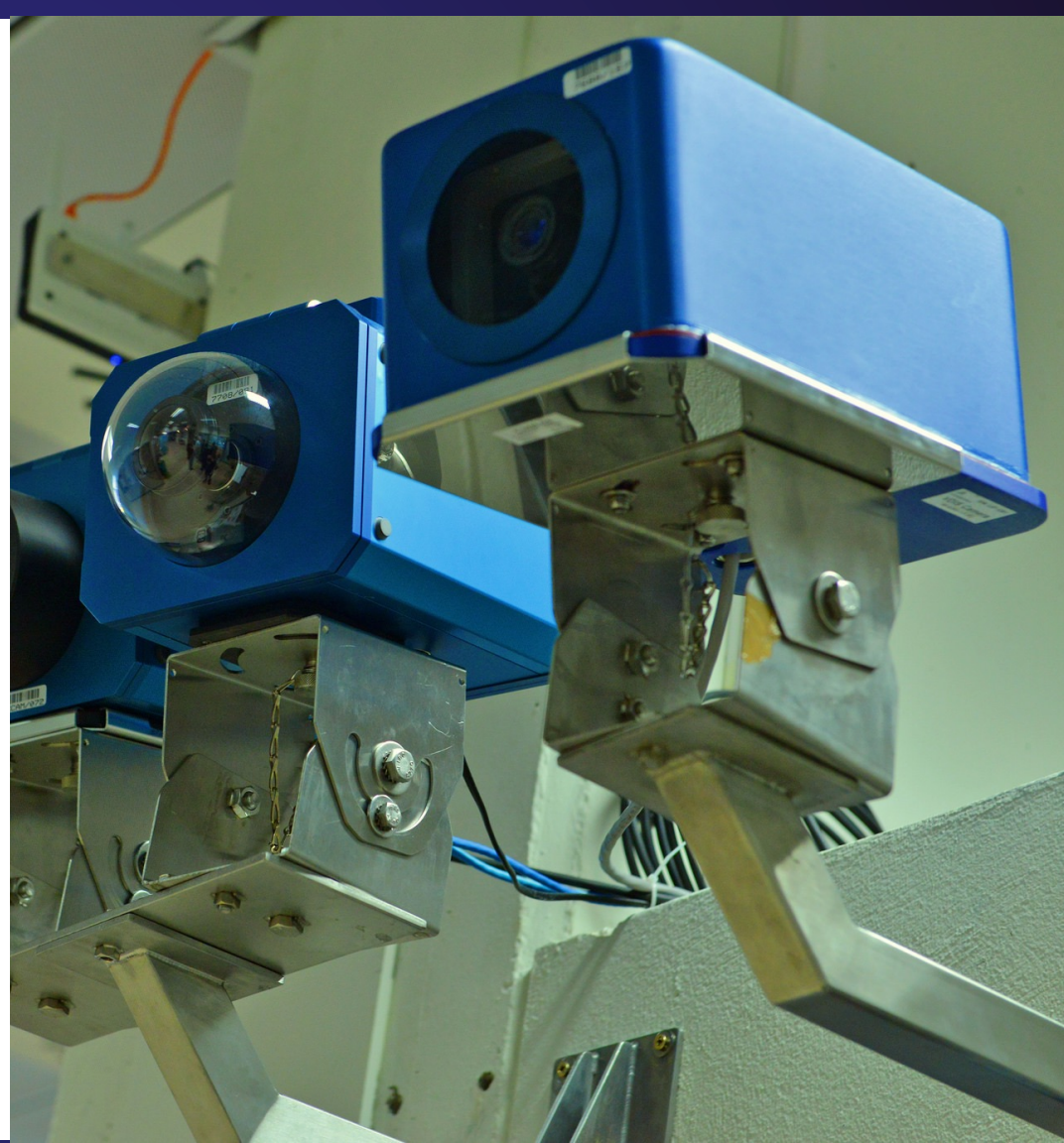




\section{Leveraging Los Alamos as a Testbed}

\section{tos:}

LANL has a wide range of unique nuclear and radiological facilities used for experiments, training, and manufacturing

The laboratory has a focused effort aimed at developing nextgeneration, dynamic sensing systems by building out testbeds at our facilities to advance the science of signatures

To that end, this work was funded internally by LANL's LaboratoryDirected Research \& Development (LDRD) Program 


\section{> Project Overview}




\section{Characterizing Activity Patterns}

The objective of safeguards is to deter the spread of nuclear weapons through early detection of the misuse of nuclear material or technology

Increased use of continuous, unattended monitoring has opened the door for the IAEA to move beyond static signatures to characterizing dynamic activities in aggregate over time and space

Characterizing activity patterns provides a baseline to define "normal" so we can identify early indicators of events and cue on things that are "abnormal"

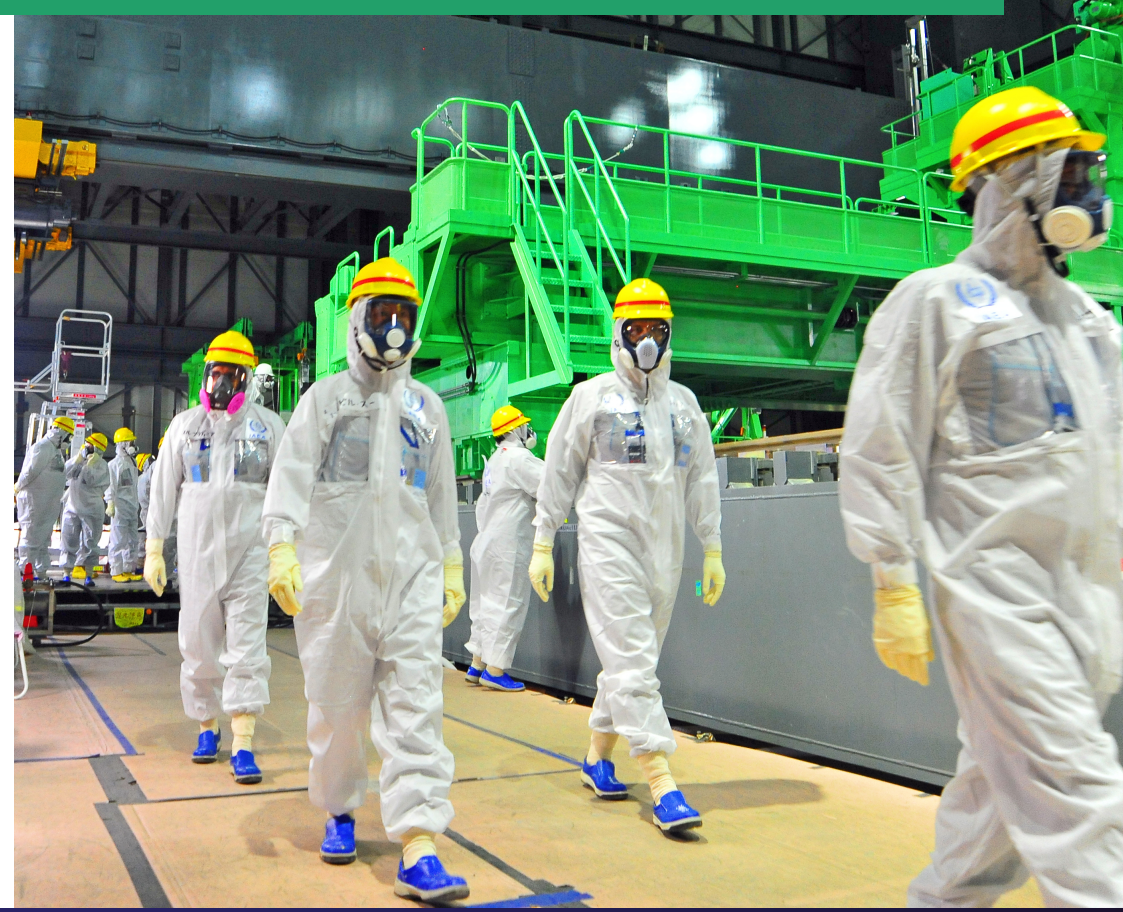




\section{Proof-of-Concept Studies}

Kendra Van Buren, Francois Hemez, Emily Casleton

How to efficiently combine data streams to automatically identify the state of operations at a facility?

Successful demonstration of disparate data integration, feature extraction, and predictive modeling of activities of interest at DARHT and PF-4

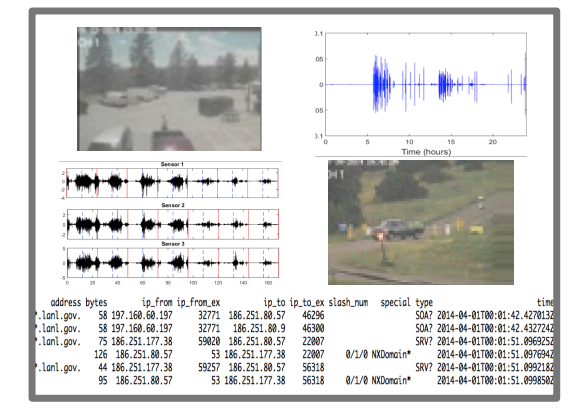

Disparate Data Streams

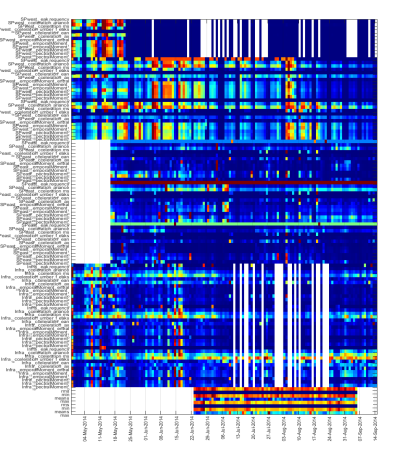

Feature Extraction

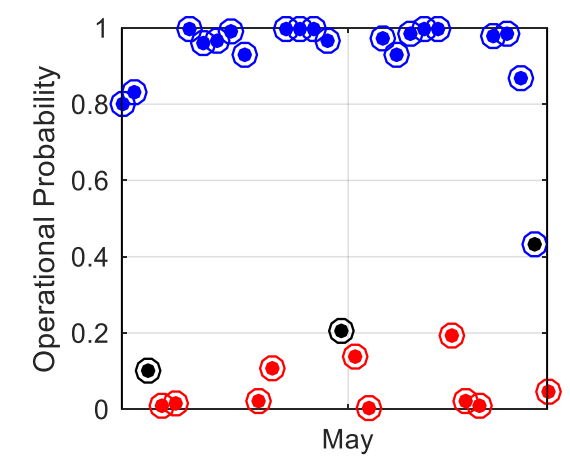

Predictive Modeling
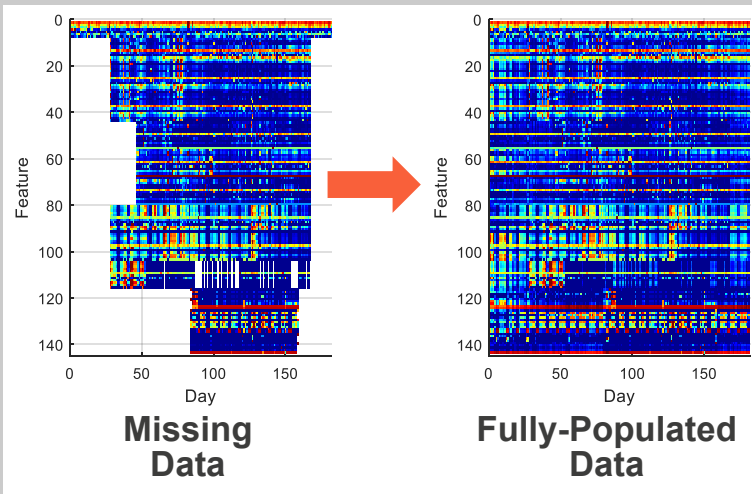

How to deal with missing or incomplete data?

Exploration of statistical methods that utilize the underlying correlation structure and track uncertainty over time 


\section{Driving Data to Decisions}

The safeguards community increasingly faces big data challenges, but data science alone is not enough

Solutions will also require domain expertise and experimental resources for validating new approaches

In this project, we have taken a multidisciplinary approach that goes beyond theory to demonstration

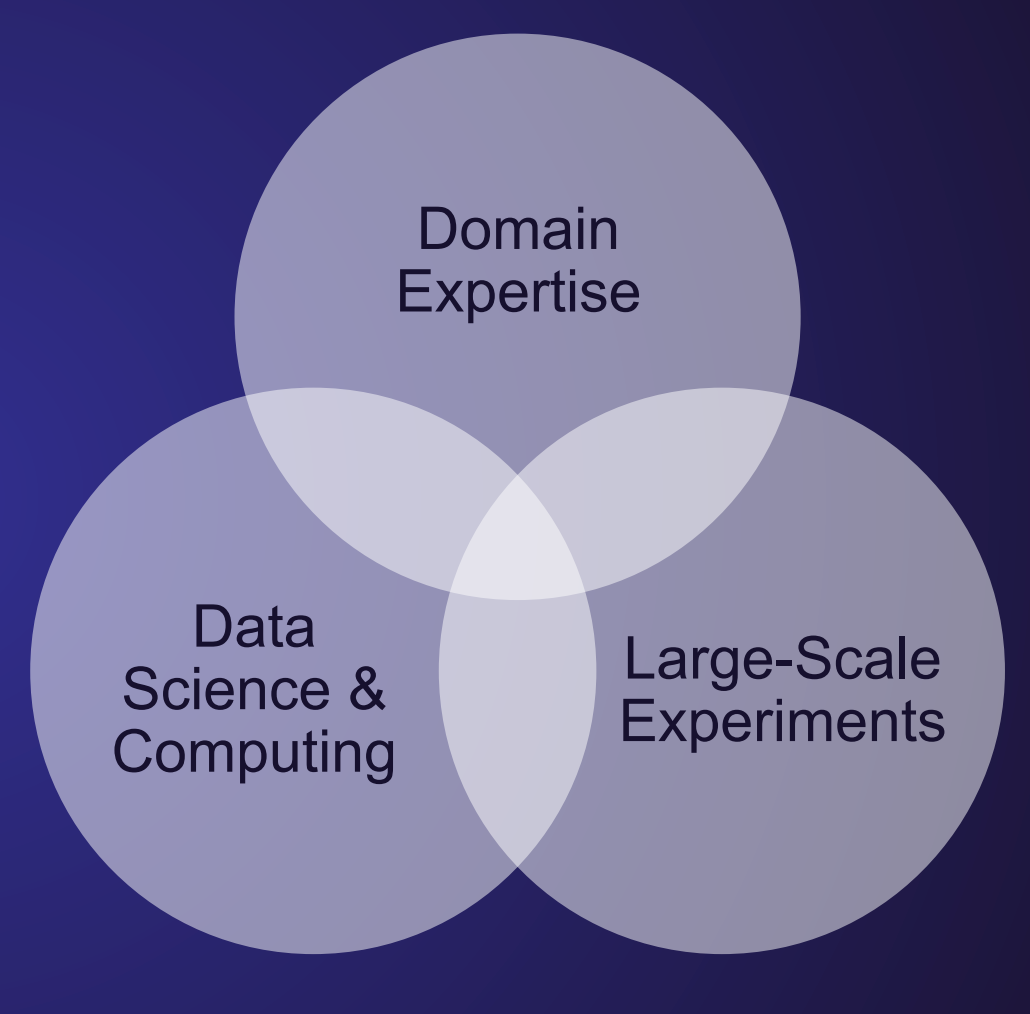




\section{FY18 Tasks}

\section{Design a sensor network for recognizing targeted activities}

- Evaluate candidate facilities, survey available equipment and data streams, design sensor network, obtain necessary approvals, procure new equipment as needed

\section{Design the associated data management architecture}

- Evaluate and implement tools for data storage and processing, bring the team into compliance with a data security plan, build out a data pipeline that facilitates automation

\section{Preform scoping measurements using available sensors}

- Install sensors, test and refine sensor locations and operating parameters

\section{Preliminary data analysis}

- Exploratory analysis and data visualization, identification of informative features, analysis of correlations between disparate data sets, classification algorithms 


\section{Multidisciplinary Research Team}

\section{Project Leaders}

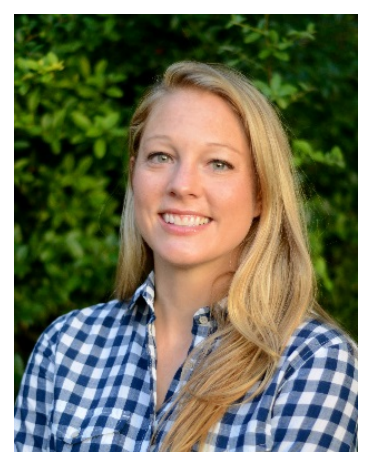

Kendra Van Buren (XCP-8) Multisource Data Fusion
Karen Miller (NEN-1)

Safeguards Technology

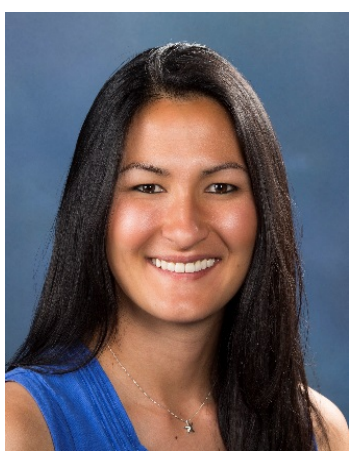

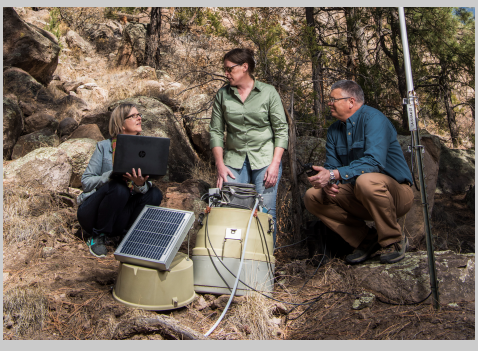
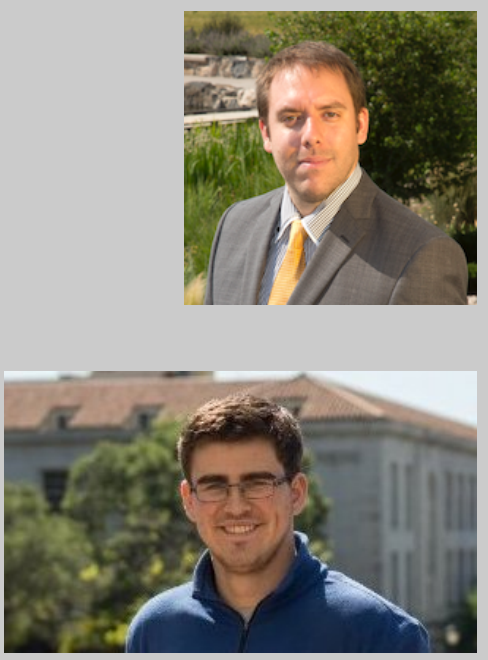

Janette Frigo (ISR-3)

Wireless Sensor Networks

Max Zeyen (CCS-7)

Computer Science

Austin Wright (student ${ }^{*}$ )

Machine Learning

*UC Berkeley 
$>$ The Facility

Los Alamos National Laboratory

UNCLASSIFIED

$10 / 9 / 18 \mid 10$ 


\section{TA-66 Safeguards Training Facility}

TA-66 is used for nondestructive assay training courses featuring hands-on $\mathrm{U}$ and $\mathrm{Pu}$ measurements

Building includes office spaces, conference rooms, and a laboratory

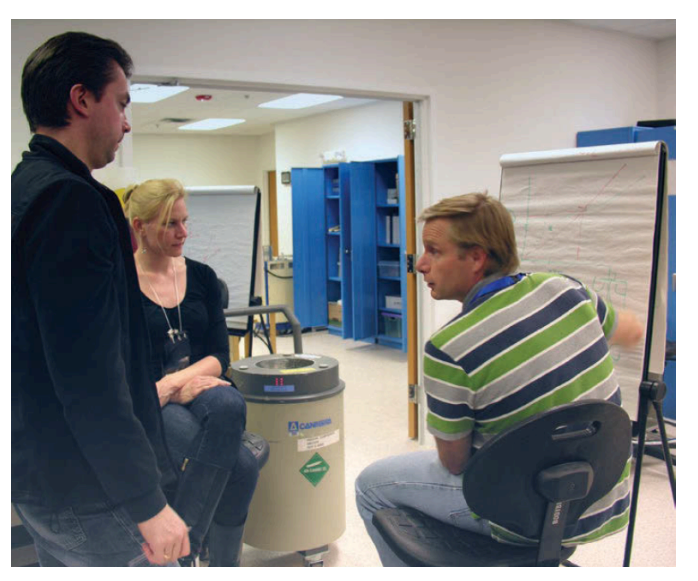

Course instructor with IAEA safeguards inspectors in the TA-66 laboratory
How Do You Find Plutonium? Go To Nuclear Inspector School

October 19, $2015 \cdot$ 4:25 AM ET

Heard on Morning Edition

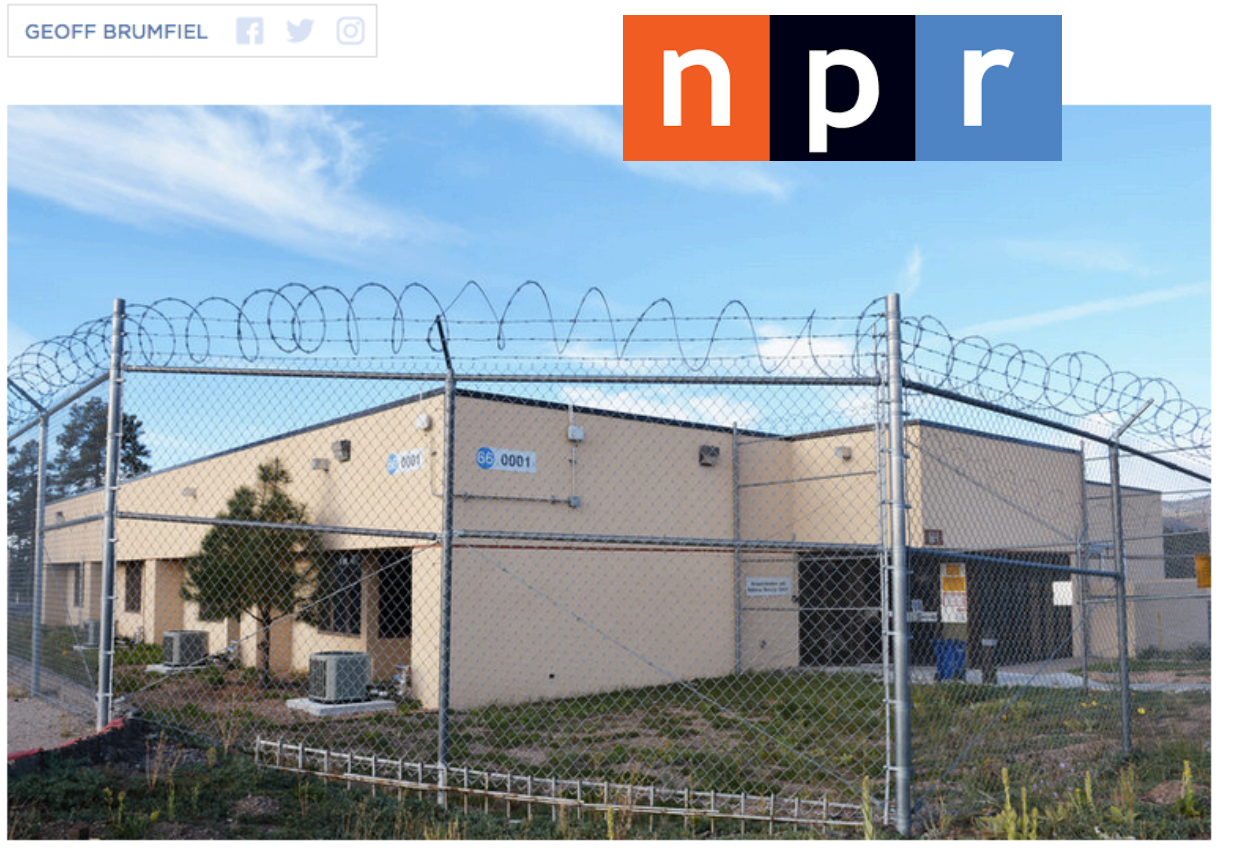




\section{Why TA-66?}

Category 3 radiological facility where sealed sources are used regularly for training and experiments - e.g., U, Pu, MOX, fresh fuel assemblies

We are taking a phased approach to the work where relatively simple operational modes aid in unambiguous initial results

The fact that it is not a production facility allows for greater flexibility in performing experiments

No sensitive operations are performed at the facility, which minimizes security overhead and allows results to be shared widely 


\section{> Data Streams}




\section{Nuclear Material Inventory Records}

\section{LANL's Source Tracker database} logs transactions associated with nuclear material movements between material balance areas

The database records information such as

- Date and time

- Transaction type (check in, check out, transfer, etc.)

- Source ID number

- Material type (U, Pu, MOX, etc.)
Number of transactions by hour of day for course days vs. non-course days at TA-66

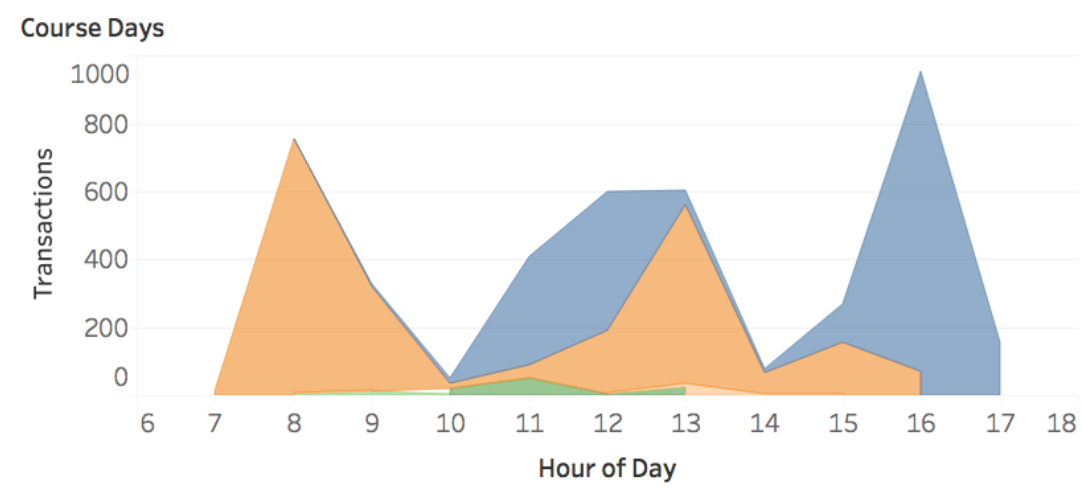

Non-Course Days

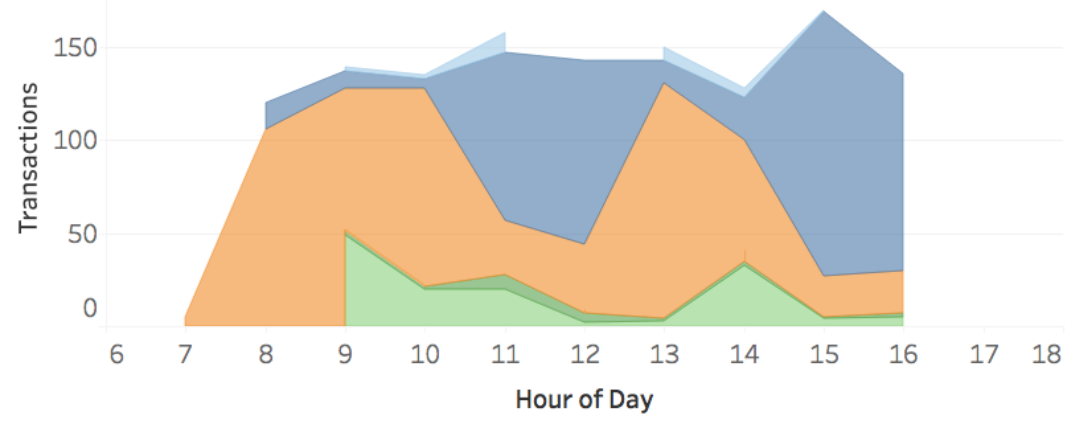




\section{Unattended Monitoring System (UMS)}

We currently have a standalone, ${ }^{3} \mathrm{He}-$ based neutron slab detector in the TA-66 laboratory collecting data continuously

Standard IAEA hardware and software

- Portable Handheld Neutron Counter (PHNC)

- Advanced Multiplicity Shift Register (AMSR)

- Laptop running Multi-Instrument Collect (MIC)

Several UMS cabinets are being installed in the laboratory now that the team plans to use once they are operational

- This effort is funded by NNSA/NA-241

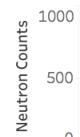

Non-Course Day
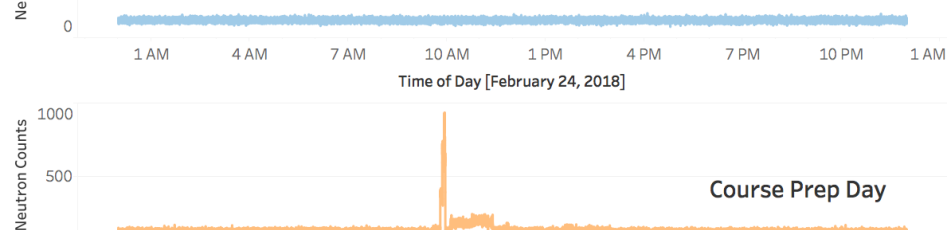

Course Prep Day
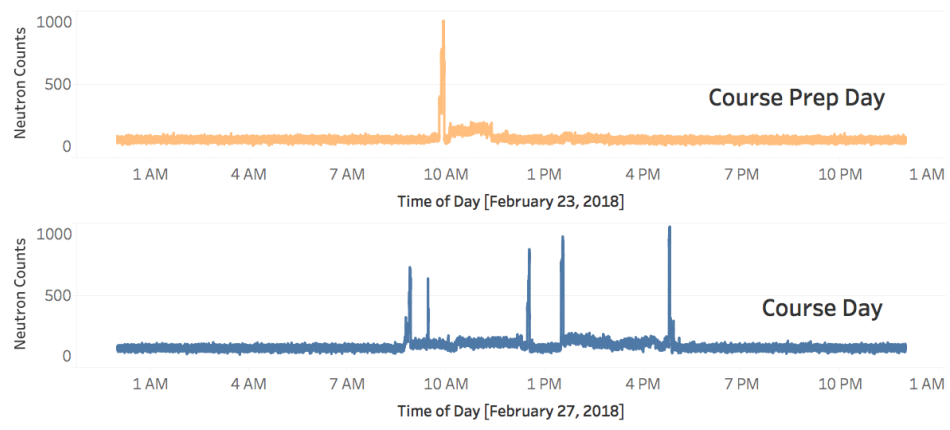

2-ft x 2-ft UMS cabinet under development for installation at TA-66

System contains a neutron coincidence counter and gamma-ray spectrometer 


\section{Custom Surveillance Sensors}

Eight inexpensive sensor nodes assembled with COTS components \& capable of onboard processing
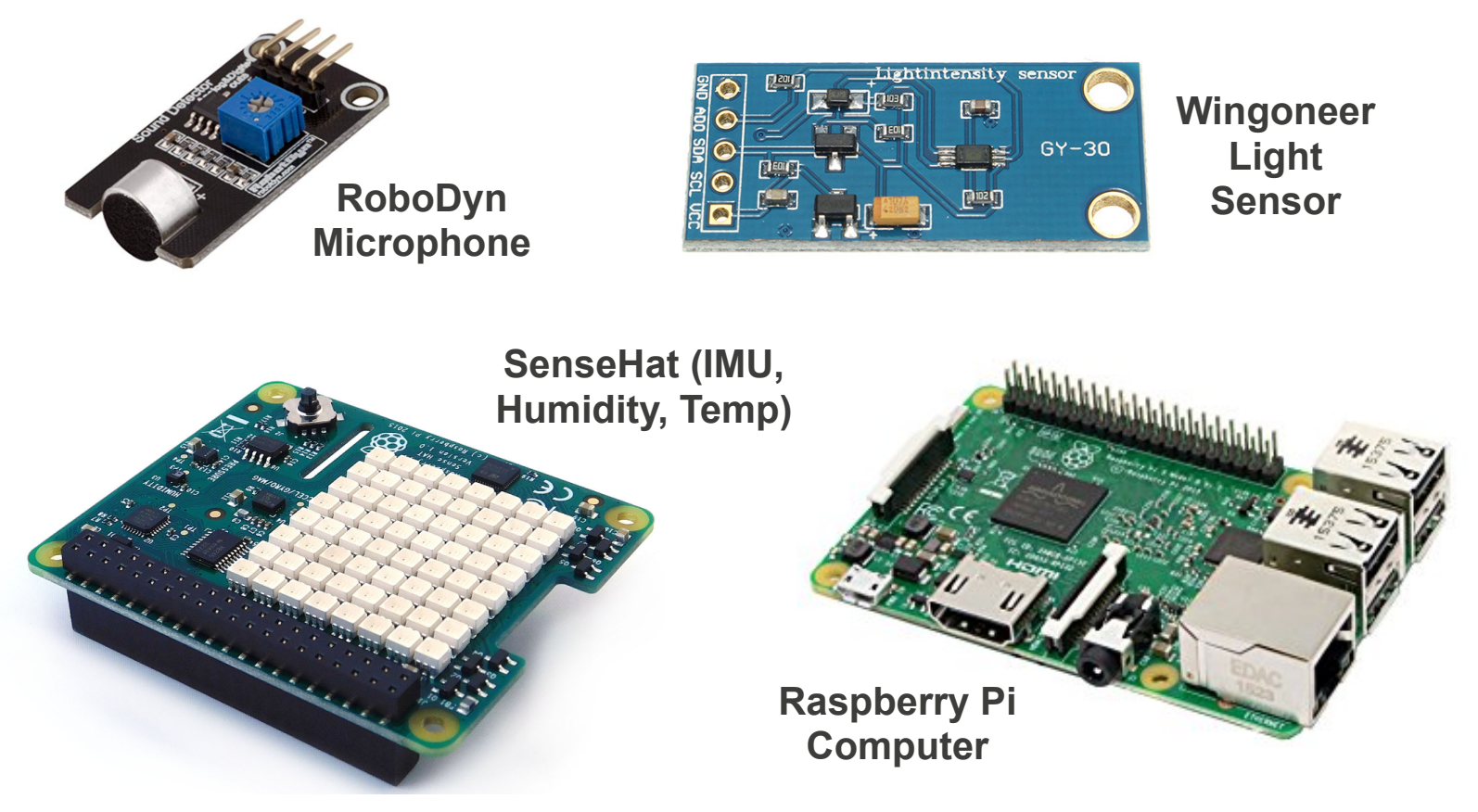

Wingoneer Light

Sensor

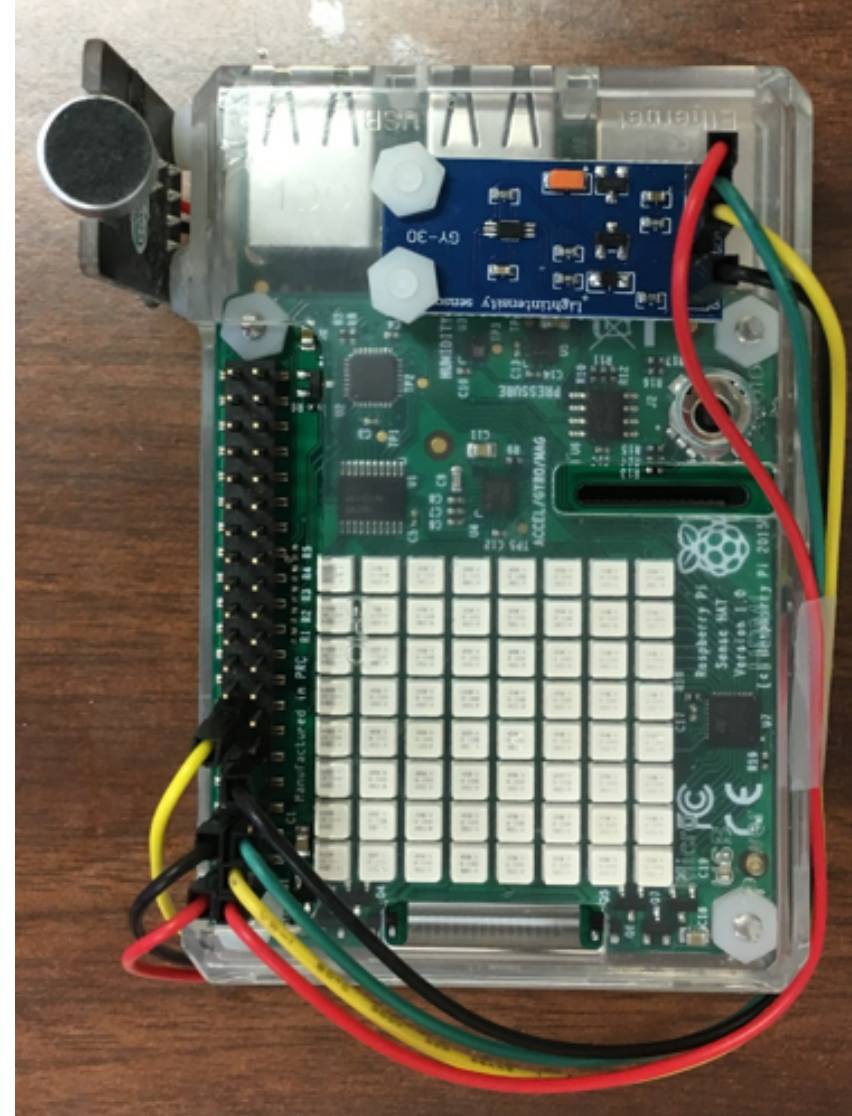




\section{Wireless Sensor Network (WSN)}

The surveillance sensors can be operated wired or wireless

The Zigbee radio has a $300-\mathrm{ft}$ range and supports wireless networks that require

- Low data transfer rate

- Energy efficiency

- Secure networking

Sensor data can hop from node-to-node to get to the base station or sink node if necessary

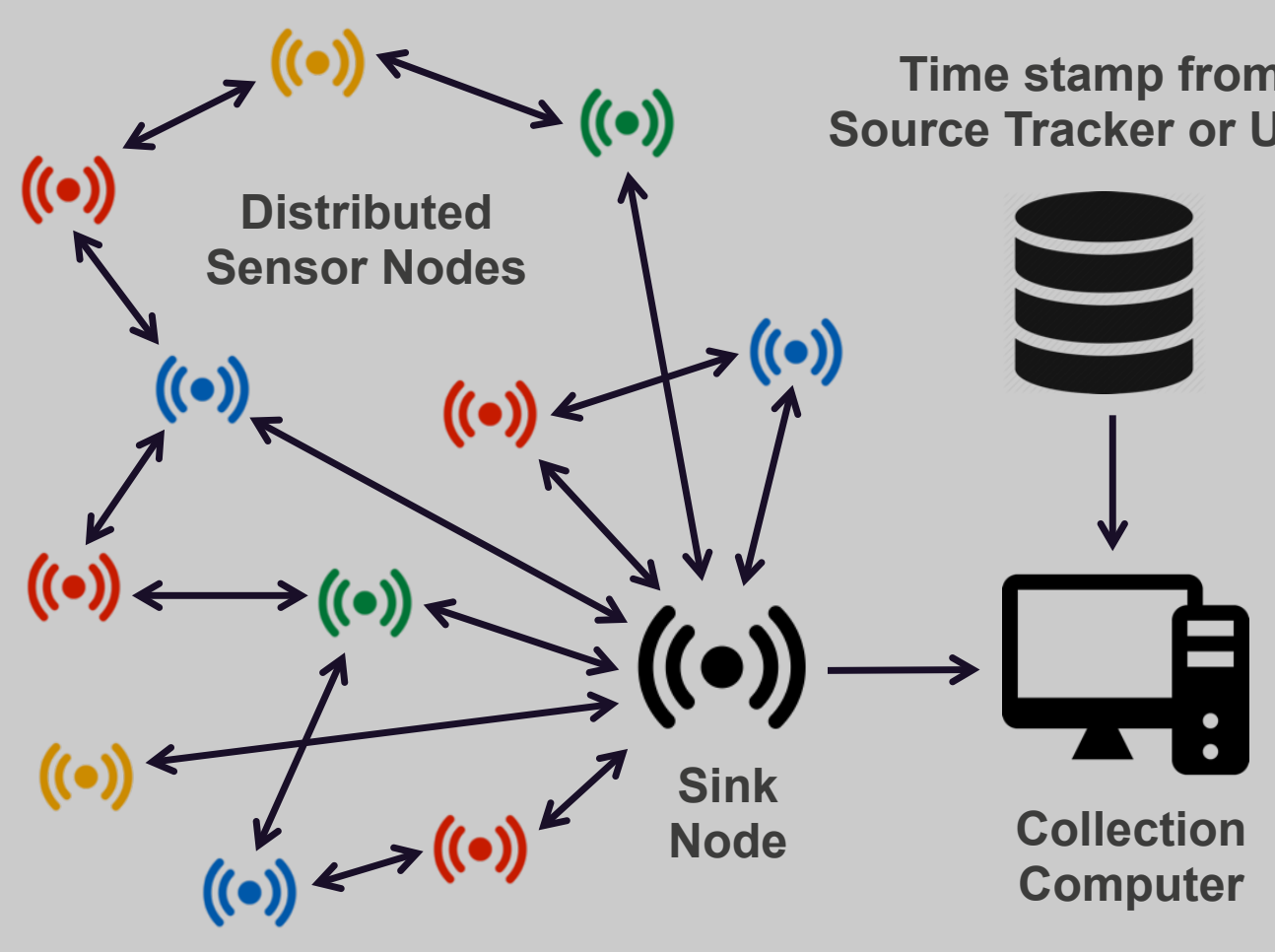

The team is collaborating with Prof. Vinod Kulathumani from West Virginia University on robust and scalable WSN protocols 


\section{$>$ Data Management}




\section{Building a Sustainable Data Pipeline}

Our data sets are large, sometimes messy, and highly heterogeneous

Designing a data architecture that is easily maintained and facilitates automation is a major thrust of this project

The first step was to set up a centralized and secure collaborative work space for the team using LANL's GitLab service

- GitLab is similar to GitHub but resides on the lab's internal networks

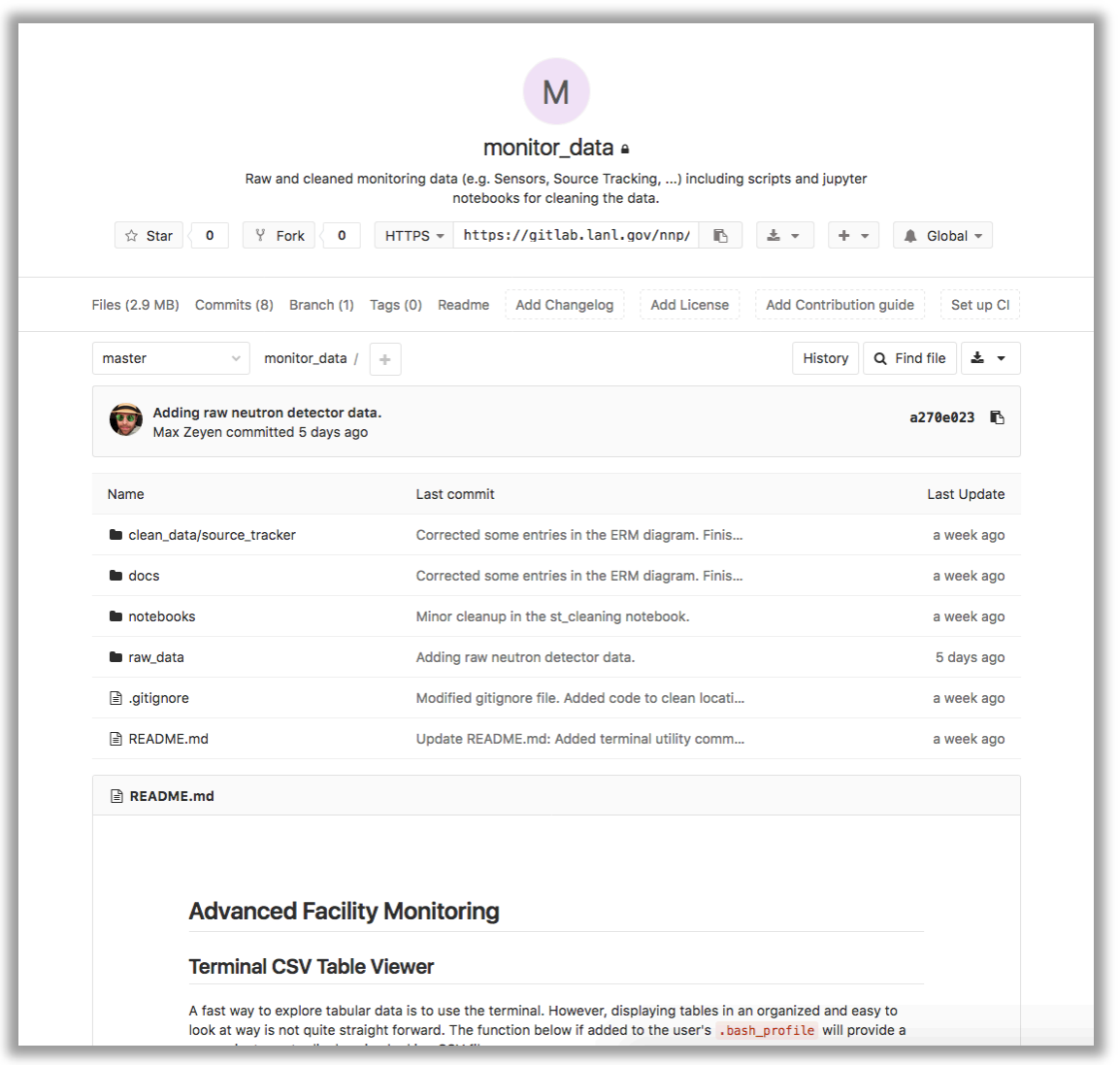




\section{Data Organization \& Storage}

Data must be organized, well-documented, consistently formatted, and error-free. Cleansing the data is often the most taxing part of data science and is frequently $\mathbf{8 0 \%}$ of the work.

— DJ Patil and Hilary Mason, "Data Driven"

Raw data is cleaned on ingest using a variety of Python scripts and Jupyter notebooks to remove corrupt entries and fields that are irrelevant for analysis

Cleaned data automatically populates a MongoDB database

- MongoDB is a free and open-source NoSQL mongo database program 


\section{Big Data Processing}

Our test environment runs on LANL's Darwin cluster

The team has a share folder on Darwin that requires user authentication for access control

We have set up a RESTful service and REST API for remote data

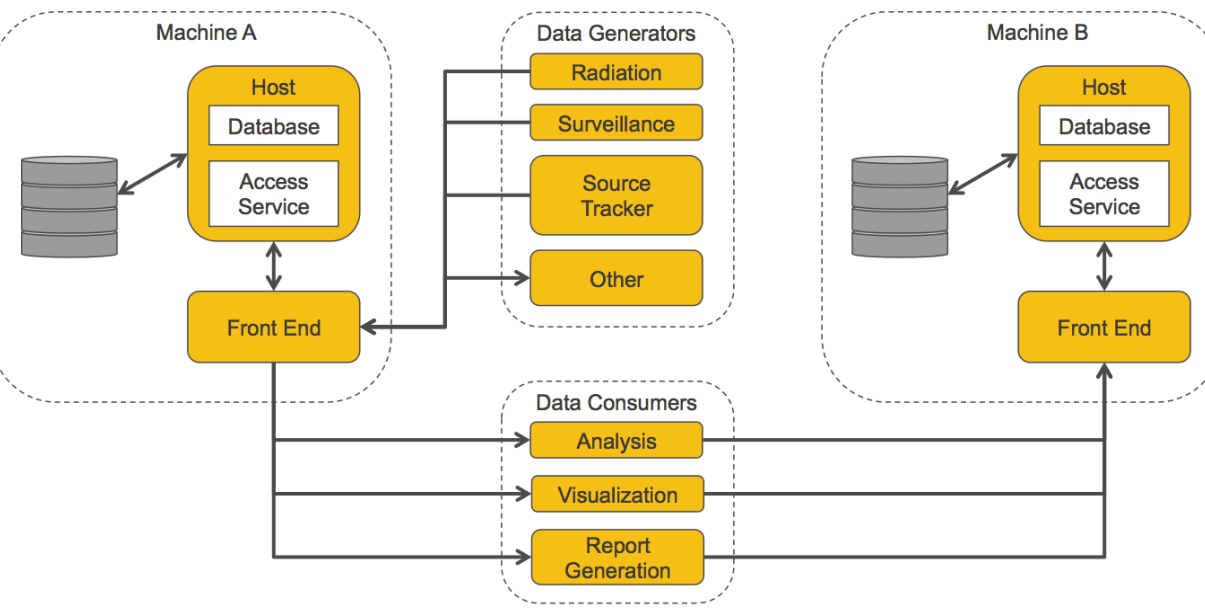
access

The configuration includes SSH tunneling to open up the Darwin front end to the data consumers

Amazon Web Services (AWS) GovCloud is a potential future option for data storage and processing that the team is currently exploring 


\section{$>$ Exploratory Analysis}





\section{Feature Extraction \& Dimensionality Reduction}

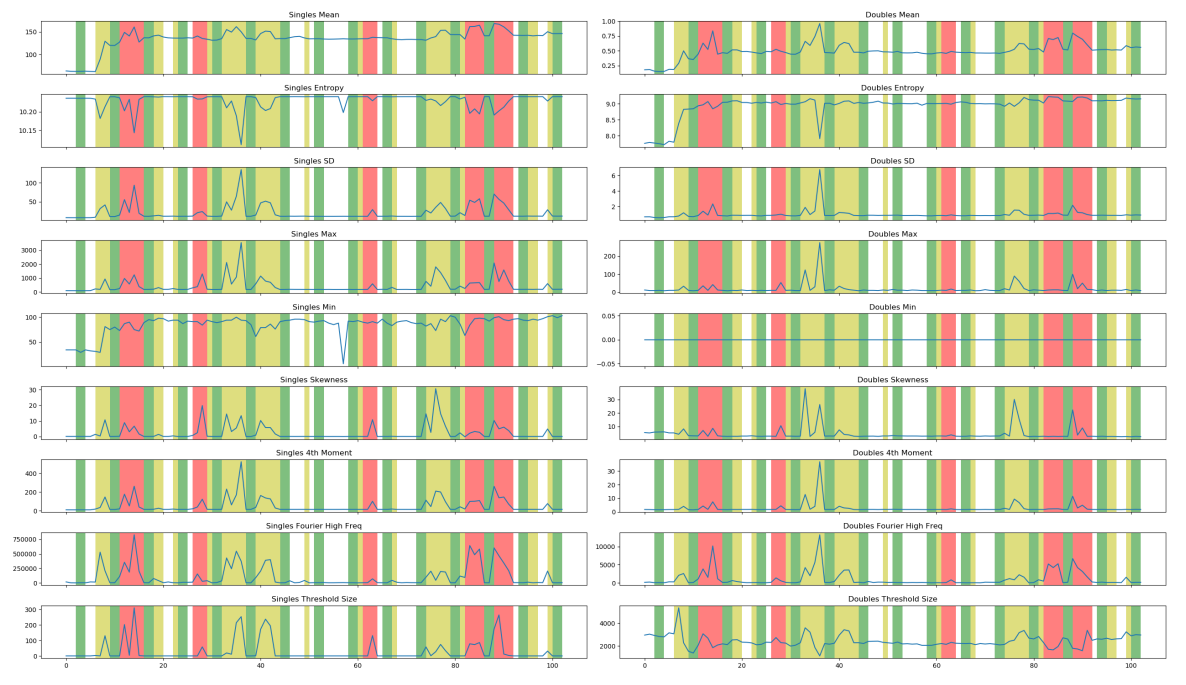

Feature Extraction

For the neutron data, we considered basic descriptive features (e.g., mean, standard deviation) as well as features that describe the spikey and highly-concentrated nature of the data
CCA Cross Decomposition with Labels

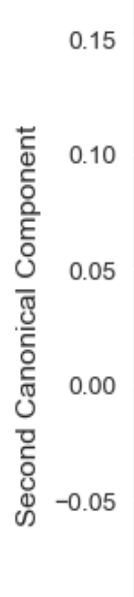

$-0.10$
$-0.2$

$-0.1$

0.0 First Canonical Component

Canonical correlation analysis of the neutron data and inventory records 


\section{Classification Models}

Once the feature space was built, we performed proof-ofconcept testing on a variety of classification models

The confusion matrices show the prediction probability for each predicted vs. true label pair

Preliminary results provide confidence that accurate models for activity characterization under realistic operational conditions can be developed
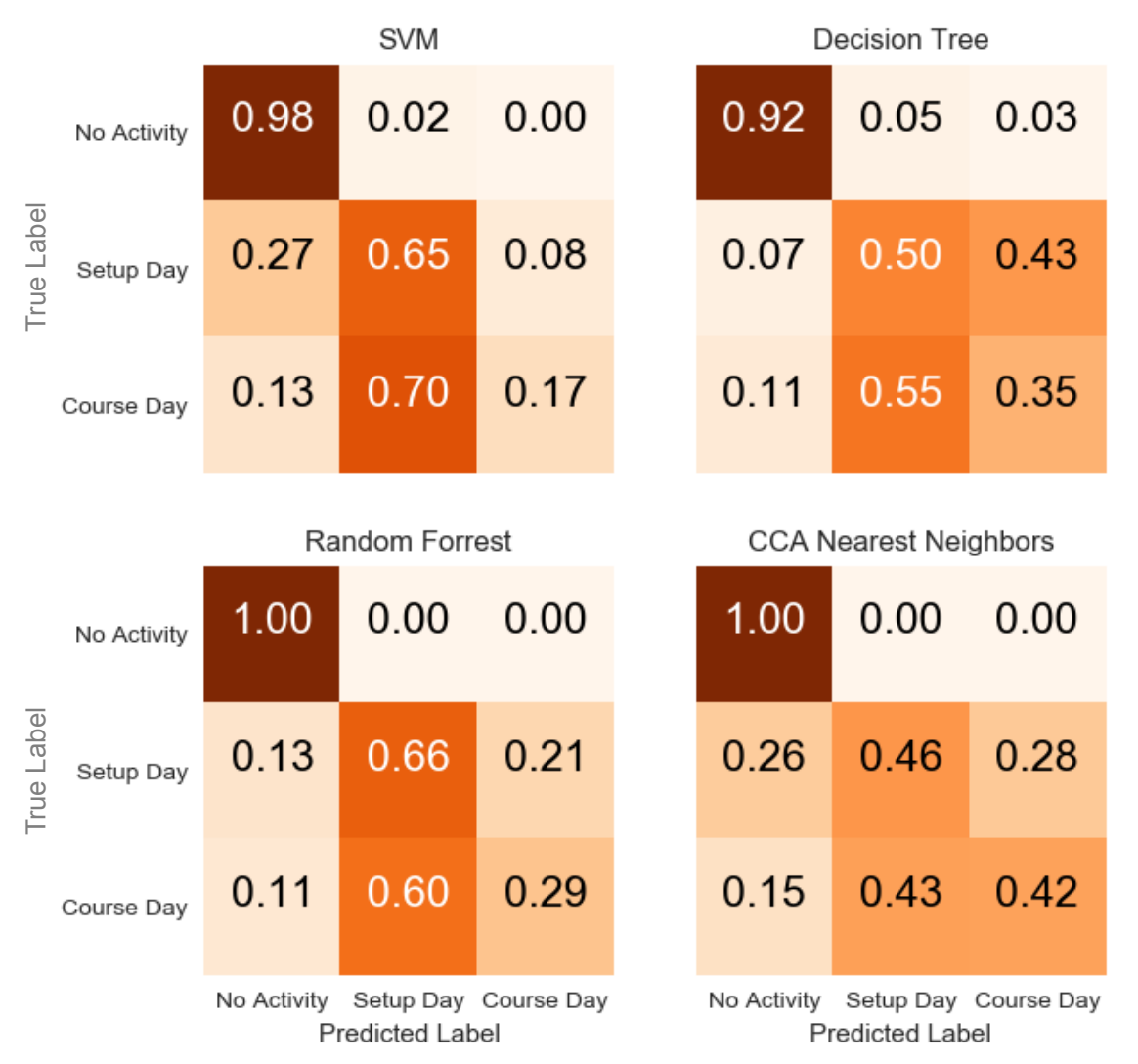

CCA Nearest Neighbors

\begin{tabular}{|c|c|c|}
\hline 1.00 & 0.00 & 0.00 \\
\hline 0.26 & 0.46 & 0.28 \\
\hline 0.15 & 0.43 & 0.42 \\
\hline
\end{tabular}




\section{Conclusions}

The convergence of safeguards technology with modern data science has the potential to generate revolutionary capability advances for the IAEA

We are exporing whether characterizing activity patterns across multisource data streams can provide the IAEA with enhanced capability to detect misuse and diversion within safeguarded facilities

This approach would represent a paradigm shift from a focus on the known to discovery of the unknown 
If you went to bed last night as an industrial company, you're going to. wake up today as a software and analytics company.

- Jeff Immelt, Former CEO General Electric 